\title{
Clinical studies of the High-Intensity Narrow-Spectrum light Environmental Decontamination System (HINS-light EDS), for continuous disinfection in the burn unit inpatient and outpatient settings
}

\author{
Sarah E. Bache ${ }^{a, b, *}$, Michelle Maclean ${ }^{b}$, Scott J. MacGregor ${ }^{b}$, John G. Anderson ${ }^{b}$, \\ George Gettinby ${ }^{c}$, John E. Coia ${ }^{d}$, Ian Taggart ${ }^{a}$ \\ a Burn Unit, Canniesburn Plastic Surgery Unit, Glasgow Royal Infirmary, Glasgow, United Kingdom \\ ${ }^{\mathrm{b}}$ The Robertson Trust Laboratory for Electronic Sterilisation Technologies (ROLEST), Department of Electronic and Electrical Engineering, \\ University of Strathclyde, Glasgow, United Kingdom \\ ${ }^{\mathrm{c}}$ Department of Mathematics and Statistics, University of Strathclyde, Glasgow, United Kingdom \\ d Department of Clinical Microbiology, Glasgow Royal Infirmary, Glasgow, United Kingdom
}

\section{A R T I C L E I N F O}

Article history:

Accepted 18 March 2011

Key words:

HINS-light

Burn

Thermal injury

Infection

Environmental decontamination

Cross-contamination

Bacteria

\begin{abstract}
A B S T R A C T
Infections are the leading cause of morbidity and mortality in burn patients and prevention of contamination from exogenous sources including the hospital environment is becoming increasingly emphasised. The High-Intensity Narrow-Spectrum light Environmental Decontamination System (HINS-light EDS) is bactericidal yet safe for humans, allowing continuous disinfection of the environment surrounding burn patients. Environmental samples were collected from inpatient isolation rooms and the outpatient clinic in the burn unit, and comparisons were then made between the bacterial contamination levels observed with and without use of the HINS-light EDS. Over 1000 samples were taken. Inpatient studies, with sampling carried out at $0800 \mathrm{~h}$, demonstrated a significant reduction in the average number of bacterial colonies following HINS-light EDS use of between $27 \%$ and $75 \%,(p<0.05)$. There was more variation when samples were taken at times of increased activity in the room. Outpatient studies during clinics demonstrated a $61 \%$ efficacy in the reduction of bacterial contamination on surfaces throughout the room during the course of a clinic $(p=0.02)$. The results demonstrate that use of the HINS-light EDS allows efficacious bacterial reductions over and above that achieved by standard cleaning and infection control measures in both inpatient and outpatient settings in the burn unit.
\end{abstract}

(C) 2011 Elsevier Ltd and ISBI. All rights reserved.

\section{Introduction}

The sequelae of burn wound infections can be devastating to the burn patient, causing progression of burn depth, graft loss, increased scarring, and subsequent sepsis, leading to multiorgan failure, and death or a significantly prolonged hospital stay. Due to advances in resuscitation and early excision regimes, it is now estimated that $75 \%$ of deaths in patients

\footnotetext{
Department to which work should be attributed: Burn Unit, Canniesburn Plastic Surgery Unit, Glasgow Royal Infirmary, 84-106 Castle Street, Glasgow G4 OSF, United Kingdom.

* Corresponding author at: Burn Unit, Canniesburn Plastic Surgery Unit, Glasgow Royal Infirmary, 84-106 Castle Street, Glasgow G4 0SF, United Kingdom. Tel.: +44 1412114324.

E-mail address: sarahbache@doctors.org.uk (S.E. Bache). 
with burns over $40 \%$ of the total body surface area (TBSA) are related to sepsis from burn wound infection or other infectious complications and/or inhalation injury [1,2]. Destruction of the skin barrier, a state of immunosuppression, and large wound areas of nutrient rich, bacteria harbouring eschar render burn patients unique in their tendency to disperse bacteria into the surrounding environment and their susceptibility to developing infections [3]. The spread of healthcare-associated infections (HAI) is an increasing worry as new strains of multi-drug resistant bacteria emerge, with a diminishing number of effective antimicrobials, leading to severe sepsis and outbreaks in burn units. Efforts to improve hand hygiene and limit the use of broad-spectrum antibiotics are important in reducing nosocomial infection rates on the burn unit, but the impact of environmental cleanliness is also becoming increasingly acknowledged [1]. The environment surrounding burn patients has been shown to be a reservoir for pathogens, and a potential source of cross-contamination between patients $[4,5]$. Bacteria surviving on inanimate surfaces for weeks or months can contaminate patients or healthcare workers, who become colonised, spreading HAI amongst patients [4-8].

Novel methods of cleaning and decontamination within hospitals have been developed, including hydrogen peroxide vapour (HPV), ultraviolet light (UV-light), and super-oxidised water [9-11]. These enable efficient temporary disinfection of the environment, but the effect is only transient and within a matter of hours the number of microorganisms begins to return to pre-decontamination levels [12]. Furthermore, they are time-consuming, requiring the removal of patients from the room, which limits their usefulness in a busy burn unit, and particularly in a burns outpatient clinic. The HighIntensity Narrow-Spectrum light Environmental Decontamination System (HINS-light EDS) is a ceiling-mounted lighting unit, which allows continuous decontamination of the clinical environment, killing bacteria through photodynamic inactivation while being safe to humans [13]. The decontamination technology uses a narrow bandwidth of visible blueviolet light, with a peak output at $405 \mathrm{~nm}$. This has previously been demonstrated in vitro to kill a wide spectrum of pathogenic bacteria, including meticillin-resistant Staphylococcus aureus (MRSA), meticillin-sensitive S. aureus (MSSA), Pseudomonas aeruginosa, Streptococcus pyogenes and Acinetobacter sp. in a dose-dependent and species-dependent fashion $[14,15]$.

The present study focused on assessing the use of the HINS-light EDS in two different burn unit environments: an isolation room housing a burn inpatient, and the burn outpatient clinic, through which several patients pass each day, so total decontamination of the room between patients is almost impossible to achieve. The propensity of burn patients to disperse pathogens into the environment means that environmental bacterial contamination is higher on the burn unit than most other hospital wards, which increases the risk of healthcare workers contaminating their hands and uniforms, and transmitting HAI to other patients in their care. This study assessed whether use of the HINS-light EDS had a significant effect on reducing the levels of environmental bacterial contamination in both the inpatient and outpatient settings, therefore potentially aiding in reducing the risk of cross-contamination of infectious pathogens from the environment to patients.

\section{Materials and methods}

\subsection{Setting}

Glasgow Royal Infirmary (GRI) has a dedicated 13-bed burn unit, arranged as six single isolation rooms, one three-bed high dependency bay, one four-bed open bay and an outpatient clinic area. Intubated patients are treated in a separate general intensive care unit. Throughout all studies, GRI burn unit infection control and isolation policies were adhered to [16]. These state that disposable gloves and aprons are donned by staff on entering isolation rooms and hands are decontaminated before and after entering the room with alcohol gel or soap and water. Appropriate ethical approval was obtained.

All air-conditioning units in the ward contain High Efficiency Particulate Air (HEPA) filters and isolation rooms are maintained at a negative pressure. Domestic staff clean inpatient isolation rooms daily, usually around $1100 \mathrm{~h}$, using chlorine-based detergents. Table tops and locker tops are wiped down periodically by nursing staff using hard surface disinfectant wipes. Following vacation of the room, a "terminal clean" is carried out. The outpatient clinic room is cleaned before the start of a clinic, around $0800 \mathrm{~h}$ by domestic staff, using chlorine-based detergents. The clinic nurse cleans the worktop, examination couch and any equipment used, using hard surface disinfection wipes between each patient.

\subsection{HINS-light EDS}

HINS-light EDS prototype units were installed in the burn unit. Two units were installed in the ceiling of two test inpatient isolation rooms and one unit in the ceiling of the smaller outpatient clinic room. Light was generated from a matrix of light-emitting diodes (LEDs), emitting a narrow bandwidth of blue-violet light centred on $405 \mathrm{~nm}$ wavelength. White LEDs are also incorporated into the HINS-light EDS such that the illumination effect is predominantly white. The HINS-light EDS units were connected to mains electricity and simply switched on and off at the wall. Minimal staff training was required and there was no disruption of the normal hospital routine. The HINS-light EDS is designed to treat an area of approximately $10 \mathrm{~m}^{2}$, with sufficient intensity to cause inactivation of exposed bacteria. Rigorous safety analysis has been carried out to standards set by the International Commission on Non-Ionizing Radiation Protection (ICNIRP) and the American Conference of Governmental Industrial Hygienists (ACGIH). It has shown that the intensity levels used in the hospital are well below the threshold limit for any adverse effects occurring, as established by ACGIH [17-19].

\subsection{Bacteriological methods}

Methods were based on previous work evaluating the efficacy of the HINS-light EDS in clinical environments [13]. Environmental bacterial samples were collected from surfaces in each 
room using $25 \mathrm{~cm}^{2}$ Baird Parker with egg yolk telurite contact agar plates (BPA plates; Cherwell Laboratories Ltd, Bicester, $\mathrm{UK})$, by the same researcher (SEB). Contact plate sampling, which enables microorganisms to be directly collected on an agar surface, was selected as the most appropriate method of assessing bacterial counts on environmental surfaces. Sample collection using broad spectrum contact agar plates, such as tryptone soya or blood agar yielded plates with too many bacterial colony forming units (cfu) to accurately enumerate in preliminary studies. Therefore, Baird Parker agar, a selective agar for staphylococcal bacteria, and an accepted marker of hospital environmental contamination in studies of hospital cleanliness, was used in the present study [20]. Staphylococci are known to survive on environmental surfaces for significant periods of time and can be transmitted between patients, staff and the environment [20,23-25]. Studies have shown the association between levels of environmental contamination with S. aureus and the size of the burn wound [21]. Furthermore, analysis of GRI burn wound swabs from the previous two years showed that MSSA and MRSA accounted for approximately $50 \%$ of all positive routine admission and twice-weekly surveillance wound cultures. It was therefore felt that an agar that selected for the commonest pathogens was justified, using the most accurate environmental sampling technique available.

Between forty and fifty sites on frequently-touched surfaces were identified around each room being studied, and bacterial samples were collected by directly pressing the contact agar plates onto the sampling site, with samples being taken from the same sites each time. After collection, contact plates were incubated at $37^{\circ} \mathrm{C}\left(98.6^{\circ} \mathrm{F}\right)$ for $48 \mathrm{~h}$ and the number of bacterial cfu on each contact agar plate was enumerated. Raw counts were statistically analysed by a chartered statistician.

\subsection{Inpatient studies}

The first part of the study was carried out in an inpatient isolation room containing a 49-year-old male, Patient A, with
45\% TBSA full thickness (third degree) flame burns, one month after admission. Routine wound surveillance swabs had isolated MRSA and P. aeruginosa, and mixed coliforms immediately before and during the study. Forty sampling sites $(n=40)$ were identified around the room (Table 1$)$. For each study, contact plate samples were collected during three phases: before the HINS-light EDS was in use (pre-HINS); after the HINS-light EDS had been on for two days (during-HINS); and after the HINS-light EDS had been switched off for a further two days (post-HINS).

Pre-HINS sampling was first carried out at $0800 \mathrm{~h}$. Immediately after this, the HINS-light EDS was switched on and remained on for $14 \mathrm{~h}$ during daylight hours, for two consecutive days. During-HINS samples were collected at $0800 \mathrm{~h}$ from the same 40 sites following this two-day use of HINS-light EDS. The HINS-light EDS was then switched off for two consecutive days, after which time post-HINS samples were collected at $0800 \mathrm{~h}$, again from the same 40 sampling sites. This study was repeated over three consecutive weeks using identical methods with the same patient in the same room but with sample collection at $1500 \mathrm{~h}$, and then $2200 \mathrm{~h}$ in order to assess the efficacy of the HINS-light EDS when samples were collected at differing times of day.

To address reproducibility, the $0800 \mathrm{~h}$ sampling protocol was repeated in rooms occupied with two further patients. Patient B was a 35-year-old female with $25 \%$ TBSA mixed deep dermal and full thickness (second and third degree) flame burn, housed in the same isolation room that Patient A had previously occupied. Her routine wound surveillance swabs had isolated MRSA and mixed coliforms. Patient C was a 55-year-old female with $40 \%$ TBSA full thickness (third degree) burn in a different room of the unit, with a mirror-image layout. Her routine wound surveillance swabs had isolated MRSA and P. aeruginosa. Ten extra sampling sites were included in the studies on Patients B and C, along both bed rails, as these two patients were bed-bound, and the bed rails were constantly upright, and an important potential site of contamination (Table 1$)(n=50)$.

Table 1 - Environmental sampling sites used in inpatient and outpatient rooms on the burn unit, with the number of samples taken from each site stated.

Inpatient isolation rooms

\begin{tabular}{|c|c|c|c|}
\hline Sampling site & No. samples & Sampling site & No. samples \\
\hline Bed sheet & 4 & Waste bin & 4 \\
\hline Locker top & 2 & Apron dispenser & 4 \\
\hline Ledge & 6 & Glove dispenser & 2 \\
\hline Table & 4 & Sink area & 6 \\
\hline Foot of bed rail & 3 & Dressings trolley & 4 \\
\hline Drip stand & 2 & Dressings shelves & 8 \\
\hline Patient chair & 2 & Worktop & 6 \\
\hline Light switches & 2 & Lamp & 2 \\
\hline Door handles & 3 & Examination couch & 6 \\
\hline Air con supply & 2 & Patient chair & 4 \\
\hline Waste bins & 4 & Power supply & 2 \\
\hline Sink area & 4 & Light switch & 1 \\
\hline Bed cot sides & 10 (Studies B and C) & Door handle & 1 \\
\hline Total & $40(50)$ & Total & 50 \\
\hline
\end{tabular}




\subsection{Outpatient studies}

Fifty sampling sites were identified on frequently touched surfaces around the outpatient clinic room $(n=50)$ (Table 1$)$. Before clinic samples were collected at $0830 \mathrm{~h}$, shortly after the room had been cleaned. Clinics ran between $0900 \mathrm{~h}$ and $1600 \mathrm{~h}$, and between seven and 12 burns patients were seen per clinic. After clinic samples were collected at $1630 \mathrm{~h}$ from the surfaces, immediately adjacent to where the 50 sites had been sampled before clinic. Samples were collected $30 \mathrm{~min}$ before and 30 min after two clinics when the HINS-light EDS was switched off (HINS off) and two clinics when the HINSlight EDS was switched on continually for $8 \mathrm{~h}$ during the clinic (HINS on).

\subsection{Statistical analysis}

The pre-HINS and post-HINS sampling periods in the inpatient room studies acted as controls for each during-HINS sampling period. A rise in the average number of bacterial $\mathrm{cfu}$ in the post-HINS samples indicated that reductions seen in during-HINS samples were not due to a general decrease in bacterial shedding by the patient over the two days, but the effect of the HINS-light EDS. For the outpatient clinic investigation, the study was repeated during two clinics in the absence of the HINS-light EDS. This acted as a control to show the expected increase in contamination levels usually seen throughout the course of a typical burns outpatient clinic. Statistical software (Minitab version 15) was used and a log-transformation was found to normalise data and equalise variances when analysing cfu data. For the inpatient studies, analysis of variance (ANOVA) and Tukey pair-wise comparisons were undertaken. The cfu counts per plate were compared between the three periods, pre-HINS, HINS and post-HINS. A 95\% confidence interval (CI) was calculated for the differences obtained between the means of the three sampling periods. For the outpatient studies, the differences in cfu count before clinic and after clinic was compared with and without the use of the HINSlight EDS. Results were displayed using mean values and statistical testing was carried out at the $5 \%$ significance level $(p \leq 0.05)$.

\section{3.}

\subsection{Inpatient studies}

Results from the five inpatient studies are summarised in Table 2. Samples collected in Patient A's room at $0800 \mathrm{~h}$ demonstrated a statistically significant reduction of $43 \%$ in the average number of Baird Parker agar isolated bacterial $c f u$ following two days of HINS-light EDS use $(p=0.043)$. After the light had been switched off for two days, bacterial numbers recovered to pre-decontamination levels, a $48 \%$ rise, $(p=0.040)$. Sample collection at $1500 \mathrm{~h}$ demonstrated a $45 \%$ reduction in bacterial contamination following two days of HINS-light EDS use, which was not statistically significant $(p=0.252)$. The study with samples collected at $2200 \mathrm{~h}$, produced a $39 \%$ reduction in the number of cfu

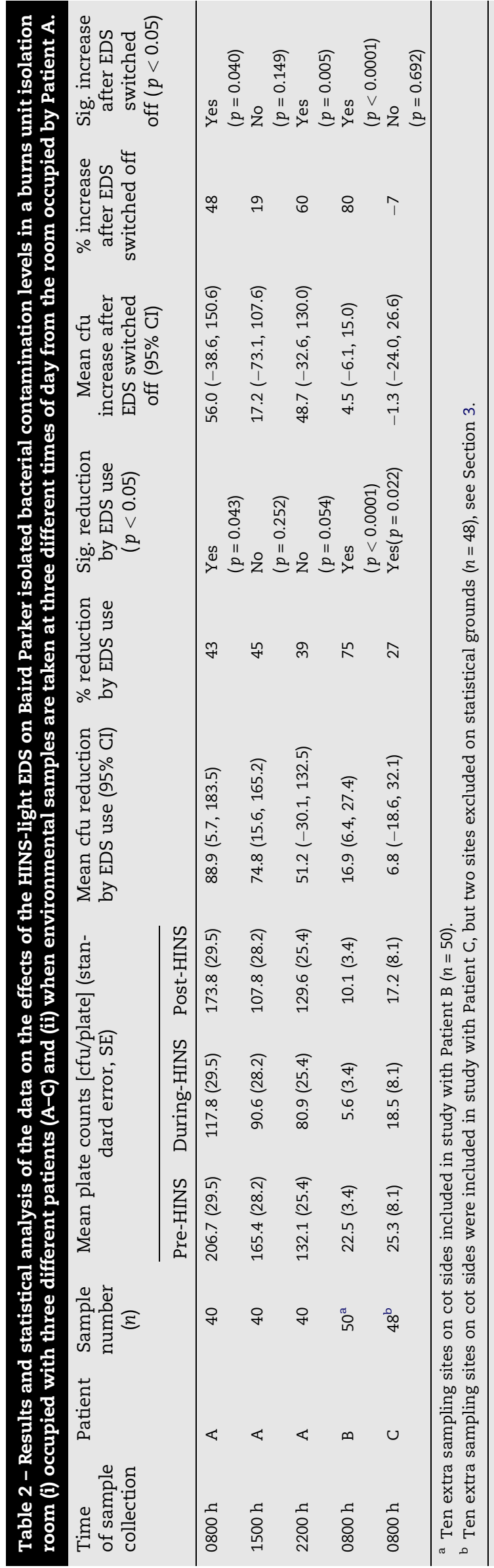


following two days of HINS-light EDS use, again not statistically significant $(p=0.054)$. After the light had been switched off again for two days there was a statistically significant $60 \%$ rise in bacterial contamination $(p=0.005)$.

The results from $0800 \mathrm{~h}$ sampling carried out in the room occupied by Patient B confirmed these findings. A significant $75 \%$ reduction in the average number of cfu was achieved following two days of HINS-light EDS use $(p<0.0001)$. When the light was switched off again, the average number of $\mathrm{cfu}$ rose by $80 \%(p<0.0001)$. In the study involving Patient $C$, the average number of bacterial cfu increased slightly from 25.2 to $25.5 \mathrm{cfu}$ following the use of the HINS-light EDS. However, the statistical analysis indicated an exceptionally unusual observation associated with the two samples from the sink site in the during-HINS sampling period. From the least squares fitted model, the standardised residual was estimated to be 8.1 and the pattern associated with the sink site was inconsistent with all other sites. A further analysis was undertaken excluding samples from the sink site $(n=48)$ and this demonstrated a significant $27 \%$ reduction from 25.3 to $18.5 \mathrm{cfu}(p=0.022)$. There was a small $(7 \%)$ decrease in the average number of $\mathrm{cfu}$ when the light was switched off again for two days, but this was not statistically significant $(p=0.692)$.

\subsection{Outpatient studies}

Results of the outpatient studies are summarised in Table 3. For studies both with and without HINS-light EDS intervention, 50 samples were collected at the start and end of two clinics. The combined results were analysed using a block design to take account of the findings from the two clinics. The difference between clinics with and without HINS-light EDS was then compared. The mean number of Baird Parker agar isolated bacterial cfu per plate before HINS off clinics was $8.1 \mathrm{cfu}$, and rose to $22.2 \mathrm{cfu}$ during the course of the clinics. This increase in contamination levels was expected, due to the dispersal of bacteria into the air and onto environmental surfaces during dressing changes and wound care of between seven and 12 patients a day. During HINS on clinics, the mean number of bacterial colonies at the start of the clinic was $6.5 \mathrm{cfu}$, and only rose to $12.0 \mathrm{cfu}$ by the end of the clinic. This indicated that the amount of additional contamination of the room, released throughout the course of a burn outpatient clinic, was reduced by an average of $8.6 \mathrm{cfu}$ per plate by the HINS-light EDS. This was the equivalent of a significant $61 \%$ efficacy $(p=0.02)$.

\section{Discussion}

The consequences of HAI for burn patients and the burn unit as a whole are serious and multiple. Prevention, identification and eradication of nosocomial infections is thus becoming an increasingly important area of burn care research [1]. The vital importance of infection control and isolating burn patients has been recognised for many decades [22]. More recently, the role of the burn unit environment in harbouring pathogens including MRSA that can survive on dry surfaces for weeks or months has been acknowledged [23-25]. A 42\% transmission rate of MRSA to the hands of healthcare workers who had no direct patient contact, as a result of touching contaminated surfaces has previously been demonstrated [26,27]. The reduction of the environmental reservoir of nosocomial infection is imperative and the current study adds further evidence of the role that the HINS-light EDS may have in achieving this.

Previously published data on the use of the HINS-light EDS as a method of decontamination for hospital inpatient environments, demonstrated reductions in the total number of environmental staphylococcal-type bacteria of between $56 \%$ and $86 \%$, when samples were collected at $0800 \mathrm{~h}$ [13]. The current study logically develops that work by investigating the reduction achieved at three different times of day, in rooms housing different burn patients, and examining specifically its use in both the inpatient and outpatient setting in one of the most important areas for infection control in the hospital: the burn unit. In the burn inpatient isolation room, the HINS-light EDS has proved to have a significant benefit in reducing environmental contamination levels by between $27 \%$ and $75 \%$ on samples taken at $0800 \mathrm{~h}$, over and above the hospital's current stringent infection control and hygiene measures. This effect was achieved with an exposure of $14 \mathrm{~h}$ a day for two consecutive days, with the light being switched off overnight, in order that it did not affect the patient's sleep.

Differences in the levels of bacterial contamination during daylight hours - likely due to direct contamination by patients or staff, or cleaning by domestic staff - is reflected on sampling at $1500 \mathrm{~h}$ and $2200 \mathrm{~h}$ when there was much more variability of activity within the room. There is no logical reason to suspect that the HINS-light EDS would be any less effective at these times of day than at $0800 \mathrm{~h}$ : indeed it might be expected that $0800 \mathrm{~h}$ sampling would produce the least dramatic reduction in contamination levels as the HINS-light EDS had been switched off overnight immediately before samples were

Table 3 - Results and statistical analysis of data on the effect of use of the HINS-light EDS on Baird Parker isolated bacterial contamination levels during burns outpatient clinics.

\begin{tabular}{|c|c|c|c|c|c|c|c|}
\hline \multirow{2}{*}{$\begin{array}{l}\text { HINS-light EDS } \\
\text { on/off during } \\
\text { clinics }\end{array}$} & \multirow{2}{*}{$\begin{array}{l}\text { Sample } \\
\text { number } \\
(n)\end{array}$} & \multicolumn{2}{|c|}{$\begin{array}{l}\text { Mean plate counts } \\
\text { (cfu/plate) }\end{array}$} & \multirow{2}{*}{$\begin{array}{l}\text { Mean increase } \\
\text { in cfu/plate } \\
\text { during clinic }\end{array}$} & \multirow{2}{*}{$\begin{array}{l}\text { Reduction in } \\
\text { increase of cfu } \\
\text { with EDS on } \\
(95 \% \text { CI })\end{array}$} & \multirow{2}{*}{$\begin{array}{l}\text { Efficacy of reduction } \\
\text { in increase of } \\
\text { cfu with EDS } \\
\text { on }(95 \% \mathrm{CI})\end{array}$} & \multirow{2}{*}{$\begin{array}{l}\text { Sig. reduction } \\
\text { in increase } \\
\text { of cfu with } \\
\text { EDS on }\end{array}$} \\
\hline & & Before clinic & After clinic & & & & \\
\hline HINS off clinics & 100 & 8.1 & 22.2 & 14.1 & $8.6(1.4,15.8)$ & $61.3 \%(10 \%, 113 \%)$ & Yes $(p=0.02)$ \\
\hline HINS on clinics & 100 & 6.5 & 12.0 & 5.5 & & & \\
\hline
\end{tabular}


taken. The main advantage to sampling at $0800 \mathrm{~h}$ is that the activity levels in the room had been relatively constant overnight before the samples were taken, as the patient was asleep in bed and staff had minimal input, preventing large surges or reductions in numbers of bacteria. This allowed a steady level of bacteria and a reliable estimate of contamination levels to be achieved when samples were taken. Although a similar pattern of reduction was demonstrated at the other times of day, there seemed to be considerable variability in staff and patient activity. This was thought to affect contamination levels and produce results that were not significant. For future studies involving environmental contamination, $0800 \mathrm{~h}$ sampling is recommended as a model to achieve the most reproducible conditions possible so that the effect of an intervention can be seen.

An incidental observation was the variability in bacterial deposition demonstrated between the three inpatients. Patient A produced higher environmental contamination, with pre-HINS levels of 206.7 cfu per plate. Patients B and C had starting populations of 22.5 and 25.3 cfu per plate respectively. There are several possible explanations for this: Patient A was ambulant around the room during the studies, although he was confined to his room. Furthermore he had loose motions on several occasions during the study, and although no infective cause for this was found, and it was assumed to be secondary to antibiotic treatment, it meant he had to go to the en-suite bathroom several times during the day and night. He had the highest \% TBSA burns, although comparable with Patient C, and all three patients had MRSA isolated from their wounds. He was also noted to have very dry flaky skin and hair, and was consequently likely to be a relatively heavy shedder of squames when compared to other patients. The exceptional counts observed for one patient at the sink location was thought to arise from gross direct contamination immediately prior to sampling. The contamination must have taken place within the room as agar plates were sealed before being removed from the room for incubation. The level of contamination may have arisen from a number of activities but none could be identified with any confidence.

The outpatient clinic was used as an example of a communal patient room in the burn unit, where it was recognised that organisms may be passed from one patient, onto a surface and thence directly to the next patient in the room. As expected, the starting numbers of bacteria were lower than in isolation rooms housing a patient constantly over long periods of time, however a significant rise in the numbers of bacteria on surfaces at the end of the clinic was seen, despite these being patients with relatively small or partly healed burns. Even though the HINS-light EDS was only on for a total of $8 \mathrm{~h}$, and the room was relatively much cleaner than the inpatient rooms to begin with, significant reductions in the increase of environmental bio burden released during a clinic were still demonstrated, with a $61 \%$ efficacy. This may lead to the use of the HINS-light EDS in other communal patient rooms, such as the physiotherapy room or bathroom, where decontamination of all surfaces is unachievable between each patient due to time limitations.

Previous studies into the bactericidal nature of $405 \mathrm{~nm}$ HINS-light have demonstrated the effect on a wide range of Gram-positive and Gram-negative organisms [15], and although levels of staphylococcal organisms were used as the marker for the current study it is important to bear in mind that levels of Gram-negative organisms will also have been reduced through use of the HINS-light EDS. The HINSlight EDS has a unique advantage in its ability to be used continuously throughout daylight hours in inpatient isolation rooms, and constantly through the day and night in other areas of the burn unit. It is efficient, simple to run, unobtrusive, and is neither dependent on staff compliance nor requires any additional staff time to implement. It must be stressed that the HINS-light EDS is not designed to replace standard cleaning routines, and the importance of wiping down surfaces, washing hands and using gloves and gowns remains. Rather, it augments current infection control methods. The HINS-light EDS is thought to have its main effect against the ubiquitous bacterial reservoirs dispersed into the air during periods of activity in the room, such as bed changes or burn dressing changes, settling on hard surfaces around the source. When surfaces are touched directly by a patient or healthcare worker, the density of organisms is more likely to be greater, so a longer exposure to the HINSlight EDS is required to decontaminate. It is probable that routine physical cleaning would take place before this, so the HINS-light EDS is not a replacement for excellent physical cleanliness in burn units, but has still been shown to maintain consistently lower levels of environmental bacteria than that achieved by physical cleaning alone.

The study of the inpatient rooms was limited in that it only examined the effect of the HINS-light EDS for a relatively short period of between $8 \mathrm{~h}$ and $14 \mathrm{~h}$ a day on two consecutive days. It is not yet known if leaving the system on for longer periods of time (for example overnight in the outpatient clinic, or at lower levels during the night in the inpatient rooms, or for more consecutive days) would continue to reduce overall levels of bacteria, or if the contamination levels would plateau after a time: this is an area of interest for future studies. Although HINS-light has wide bactericidal activity, as demonstrated in vitro $[14,15]$, this study focused on the reduction of staphylococcal type organisms, which account for over $50 \%$ of wound contaminations and infections in the GRI burn unit and give an indication of organisms which have originated from a human source, and are thus potential pathogens. While the experiment could be repeated using an agar that would allow estimation of total viable counts of all bacteria, the large number of cfu arising from some surfaces would also make accurate enumeration very difficult. Future work may address the impact of the HINS-light EDS on Gram-negative organisms, by sampling using an agar that selects for Gram-negatives alone. Further laboratory studies on the effect of the HINSlight EDS on bacteria subject to various stressing factors, or the formation of biofilms would also be of interest.

These studies provide convincing evidence that this novel technology achieves a reduction in environmental contamination levels. To demonstrate that this translates into a reduction in colonisation and infection in burn patients, in the context of the huge numbers of variables in the patients, burns and treatment administered, would be the ideal next stage, but would probably require a multi-centre trial over months or years. Such difficulties account for the paucity of evidence that many other established infection control methods and 
disinfection technologies have achieved reductions in infection rates. Rather, a logical and pragmatic approach has been adopted that a cleaner environment and cleaner hands are likely to result in the transfer of fewer numbers of bacteria to patients, and thus generate fewer infections. The impact of surface disinfection in hospitals cannot be dismissed due to the lack of outcome trials, as HAI as an outcome has reasonably low frequency, so any potential trial would suffer from low statistical power $[28,29]$.

The findings of this work provide evidence that the HINSlight EDS is an effective treatment for the reduction of environmental bacterial contaminants in different clinical situations on the burn unit. The percentage reduction observed for counts taken at different times during the day were broadly comparable for the room containing the same patient. In contrast, the percentage reduction at the same time of day for rooms housing different patients varied considerably. This is not unexpected, as contamination levels are known to differ depending on the patient, the size of burn and the patient environment [30]. A total of 34 different burn patients were treated in the outpatient clinic room, yet the presence of the HINS-light EDS in the room while they were being treated significantly reduced the environmental bacterial contamination they produced. These results suggest that for burn patients, the HINS-light EDS can potentially make an important additional contribution to the reduction of nosocomial infections which originate from transmission of pathogens from the environment, by significantly reducing the contamination of the surrounding environment.

\section{Conflict of interest statement}

The intellectual property rights of the HINS-light EDS belong to the University of Strathclyde. As co-inventors, MM, SJM and JGA have a share of the intellectual property rights. SEB, GG JEC and IT have no claim to intellectual property. The University has made all HINS-light EDS for research purposes only and no commercial company manufactures or sells this technology.

\section{Acknowledgements}

The first author would like to thank Mr Stuart Watson, Consultant Plastic Surgeon at the Canniesburn Plastic Surgery Unit, for guidance and support during the study. All authors would like to thank the Stephen Forrest Trust, the Burn Unit Fund, the Robertson Trust and the University of Strathclyde for the funding that has made this research possible. Thanks also go to Professor Gerry Woolsey for his invaluable contribution to the development and safety analysis of the system, and the staff and patients on the burns unit at GRI for their co-operation and support.

\section{REFEREN C E S}

[1] Rafla K, Tredget EE. Infection control in the burn unit. Burns 2010. doi: 10.1016/j.burns.2009.06.198.
[2] Taneja N, Emmanuel R, Chari PS, Sharma M. A prospective study of hospital-acquired infections in burn patients at a tertiary care referral centre in North India. Burns 2004;30:665-9.

[3] Polavarapu N, Ogilvie MP, Panthaki ZJ. Microbiology of burn wound infections. J Craniofac Surg 2008;19(4): 899-902.

[4] Talon D. The role of the hospital environment in the epidemiology of multi-resistant bacteria. J Hosp Infection 1999;43:13-7.

[5] Zanetti G, Blanc DS, Federli I, Raffoul W, Petignat C, Maravic $\mathrm{P}$, et al. Importation of Acinetobacter baumannii into a burn unit: a recurrent outbreak of infection associated with widespread environmental contamination. Infect Control Hosp Epidemiol 2007;28:723-5.

[6] Jawad A, Snelling AM, Heritage J, Hawkey PM. Exceptional desiccation tolerance of Acinetobacter radioresistens. J Hops Infect 1998;39:235-40.

[7] Hirai Y. Survival of bacteria under dry conditions; from a viewpoint of nosocomial infection. J Hosp Infect 1991; 19:191-200.

[8] Bonilla HF, Zervos MJ, Kauffman CA. Long-term survival of vancomycin-resistant Enterococcus faecium on a contaminated surface. Infect Control Hosp Epidemiol 1996;17(12):770-1.

[9] Boyce JM, Havill NL, Otter JA, McDonald LC, Adams NM, Cooper $\mathrm{T}$, et al. Impact of hydrogen peroxide vapour room decontamination on Clostridium difficile environmental contamination and transmission in a healthcare setting. Infect Control Hosp Epidemiol 2008; 29(8):723-9.

[10] Sweeney CP, Dancer SJ. Can hospital computers be disinfected using a hand-held UV light source? J Hosp Infection 2009;72(1):92-4.

[11] Clark J, Barrett SP, Rogers M, Stapleton R. Efficacy of superoxidized water fogging in environmental decontamination. J Hosp Infection 2006;64:386-90.

[12] Dettenkofer M, Spencer RC. Importance of environmental decontamination-a critical view. J Hosp Infection 2007;65(S2):55-7.

[13] Maclean M, MacGregor SJ, Anderson JA, Woolsey GA, Coia JE, Hamilton K, et al. Environmental decontamination of a Hospital Isolation Room using High-Intensity NarrowSpectrum Light (HINS-light). J Hosp Infect 2010; 76:247-51.

[14] Maclean M, MacGregor SJ, Anderson JG, Woolsey G. Highintensity narrow-spectrum light inactivation and wavelength sensitivity of Staphylococcus aureus. FEMS Microbiol Lett 2008;285:227-32.

[15] Maclean M, MacGregor SJ, Anderson JG, Woolsey G. Inactivation of bacterial pathogens following exposure to light from a 405-nanometer light-emitting diode array. Appl Environ Microbiol 2009;75(7):1932-7.

[16] Infection control homepage, NHS Greater Glasgow and Clyde. http://www.nhsggc.org.uk/content/ default.asp?page=home_infectioncontrol.

[17] International Commission on Non-Ionizing Radiation Protection (ICNIRP). Guidelines on limits of exposure to optical radiation from 0.38 to $3.9 \mathrm{~mm}$. Health Phys 1997;73:539-54.

[18] International Commission on Non-Ionizing Radiation Protection (ICNIRP). Guidelines on limits of exposure to ultraviolet radiation of wavelengths between $180 \mathrm{~nm}$ and 400 nm (Incoherent Radiation). Health Phys 2004; 87:171-86.

[19] American Conference of Governmental Industrial Hygienists (ACGIH). Threshold limit values (TLVs) \& biological exposure indices. Cincinnati: Signature Publications; 2007. 
[20] Dancer SJ, White L, Robertson C. Monitoring environmental cleanliness on two surgical wards. Int J Environ Health Res 2008;18:357-64.

[21] Hambraeus A. Studies on transmission of Staphylococcus aureus in an isolation ward for burned patients. J Hyg 1973;71:171-83.

[22] Watson J. The control of infection in a burns unit. J Royal College Surg Ed 1966;11(4):303-6.

[23] Boyce JM. Environmental contamination makes an important contribution to hospital infection. J Hops Infect 2007;65(S2):50-4.

[24] Talon D. The role of the hospital environment in the epidemiology of multi-resistant bacteria. J Hosp Infect 1999;43:13-7.

[25] Weber J, McManus A. Infection control in burn patients. Burns 2004;30:A16-24.
[26] Boyce JM, Potter-Bynoe G, Chenevert C, King T. Environmental contamination due to methicillinresistant Staphylococcus aureus: possible infection control implications. Infect Control Hosp Epidemiol 1997; 18:622-7.

[27] Noskin GA, Stosor V, Cooper I, Peterson LR. Recovery of vancomycin-resistant enterococci on fingertips and environmental surfaces. Infect Control Hosp Epidemiol 1995;16(10):577-81.

[28] Baillie J. Deep cleaning-valid or publicity stunt? Health Estate 2008;62(3):25-9.

[29] Fraise AP. Decontamination of the environment. J Hosp Infection 2007;65(S2):58-9.

[30] Church D, Elsayed S, Reid O, Winston B, Lindsay R. Burn wound infections. Clin Microbiol Rev 2006;19(2): 403-34. 